\title{
Characteristics of circular RNA expression in lung tissues from mice with hypoxia-induced pulmonary hypertension
}

\author{
JIAN WANG ${ }^{1,2 *}$, MENG-CHAN ZHU $^{2 *}$, BILL KALIONIS $^{3,4}$, JUN-ZHEN WU $^{1}$, LIN-LIN WANG ${ }^{2}$, HAI-YAN GE $^{5}$, \\ CUI-CUI CHEN ${ }^{2}$, XIAO-DAN TANG ${ }^{5}$, YUAN-LIN SONG ${ }^{2}$, HONG HE $^{6}$ and SHI-JIN XIA ${ }^{1 *}$ \\ ${ }^{1}$ Department of Geriatrics, Shanghai Institute of Geriatrics, Huadong Hospital, Fudan University, \\ Shanghai 200040; ${ }^{2}$ Department of Pulmonary Medicine, Zhongshan Hospital, Fudan University, Shanghai 200030, \\ P.R. China; ${ }^{3}$ Pregnancy Research Centre, Department of Maternal-Fetal Medicine; ${ }^{4}$ University of Melbourne \\ Department of Obstetrics and Gynaecology, Royal Women's Hospital, Parkville, Victoria 3052, Australia; \\ ${ }^{5}$ Department of Pulmonary Medicine, Huadong Hospital, Fudan University, Shanghai 200040; \\ ${ }^{6}$ Department of Anesthesiology, Fudan University Shanghai Cancer Center; Department of \\ Oncology, Shanghai Medical College, Fudan University, Shanghai 200032, P.R. China
}

Received March 24, 2018; Accepted June 20, 2018

DOI: $10.3892 /$ ijmm.2018.3740

\begin{abstract}
Pulmonary hypertension (PH) is a life-threatening lung disease, characterized by an increase in pulmonary arterial pressure caused by vasoconstriction and vascular remodeling. The pathogenesis of $\mathrm{PH}$ is not fully understood, and there is a lack of potential biomarkers for the diagnosis and treatment of patients with PH. Non-coding RNAs with a characteristic covalently closed loop structure, termed circular RNAs (circRNAs), are present in a number of pulmonary diseases. To the best of our knowledge, the present study is the first to use microarray analysis to determine the expression profile of circRNAs in lung tissues from mice with hypoxia-induced $\mathrm{PH}$. In total, 23 significantly upregulated and 41 significantly downregulated circRNAs were identified. Of these, 12 differentially expressed circRNAs were selected for further validation using reverse transcription-quantitative polymerase chain reaction. Putative microRNAs (miRNAs) that bind to the dysregulated circRNAs were predicted. Subsequently, bioinformatics tools were used to construct circRNA-miRNA-mRNA
\end{abstract}

Correspondence to: Dr Shi-Jin Xia, Department of Geriatrics, Shanghai Institute of Geriatrics, Huadong Hospital, Fudan University, 221 Yanan West Road, Shanghai 200040, P.R. China E-mail: xiashijinhd@163.com

Dr Hong He, Department of Anesthesiology, Fudan University Shanghai Cancer Center; Department of Oncology, Shanghai Medical College, Fudan University, 270 Dongan Road, Shanghai 200032, P.R. China

E-mail: hyc_hong@163.com

*Contributed equally

Key words: circular RNAs, pulmonary hypertension, hypoxia, microarray networks for the two most promising circRNAs, namely mmu_circRNA_004592 and mmu_circRNA_018351. Gene Ontology and Kyoto Encyclopedia of Genes and Genomes analyses of target genes of the dysregulated circRNAs revealed that these dysregulated circRNAs may serve an important role in the pathogenesis of hypoxia-induced PH. Therefore, these dysregulated circRNAs are candidate diagnostic biomarkers and potential therapeutic targets for $\mathrm{PH}$.

\section{Introduction}

Pulmonary hypertension (PH) is a complex and lifethreatening type of lung disease, which is defined by a mean pulmonary arterial pressure of $\geq 25 \mathrm{mmHg}$ at rest as measured by right heart catheterization (1). Recently, $\mathrm{PH}$ was clinically classified into five groups: Pulmonary arterial hypertension, $\mathrm{PH}$ due to left heart disease, $\mathrm{PH}$ due to lung diseases and/or hypoxia, chronic thromboembolic $\mathrm{PH}$, and $\mathrm{PH}$ with unclear and/or multifactorial mechanisms (2). Hypoxia-induced $\mathrm{PH}$, which is the focus of the present study, results from hypoxic pulmonary vasoconstriction, polycythemia and pulmonary arterial remodeling (3). Pathological changes in patients with hypoxia-induced PH manifest as dysfunction of smooth muscle cells, fibroblasts and pulmonary artery endothelial cells, which are associated with progressive obliteration of the pulmonary arteries $(4,5)$. Several pathways participate in the complex process of hypoxia-induced $\mathrm{PH}$; however, the underlying mechanism is not well-defined. Furthermore, the current treatment strategies available are limited and the prognosis is poor for patients with PH (6). Thus, novel strategies for the diagnosis and treatment of patients with hypoxia-induced $\mathrm{PH}$ are urgently required.

Circular RNAs (circRNAs) are a novel class of the non-coding RNA family that feature a covalently closed loop structure without a 5'-terminal cap or 3'-terminal poly(A) tail (7). Initially, circRNAs were considered to be functionless byproducts of the normal splicing processing. With the 
advent of next-generation sequencing, it was revealed that circRNAs were widely distributed stable entities in various cell types and were expressed in species-, tissue-, disease- and developmental stage-specific manners $(8,9)$. To date, the functions of circRNAs have not been fully elucidated. The most widely studied function of circRNAs is their ability to function as miRNA sponges. The representative circRNA, ciRS-7, has $>70$ conventional binding sites, which bind to miR-7 to decrease its expression levels and further increase the levels of miR-7-targeted transcripts (10). CircRNA located in the nucleus may participate in the regulation of transcription and alternative splicing (11). Furthermore, a number of circRNAs have been demonstrated to be translated into proteins. Legnini et al (12) firstly demonstrated that circ-ZNF609 could be translated into a small protein that specifically controlled the myoblast proliferation. These unique features of circRNAs have brought a new perspective to our understanding of the biological mechanisms underlying various diseases, including cardiovascular diseases, cancer, neurological disorders and diabetes, and the possibility of novel diagnostic biomarkers and innovative strategies for treatment (13-16). However, the role of circRNAs in hypoxia-induced $\mathrm{PH}$ remains unknown.

In the present study, a murine model of hypoxia-induced $\mathrm{PH}$ was established. To the best of our knowledge, circRNA microarray analysis was used for the first time to determine the differential expression profile of circRNAs in the lungs of mice with hypoxia-induced PH compared with the control mice. A total of 12 candidate circRNAs were selected for further validation by reverse transcription-quantitative polymerase chain reaction (RT-qPCR). Bioinformatics analyses identified the potential functions of the candidate circRNAs in the mechanism of hypoxia-induced PH. These candidate circRNAs have the potential to function as diagnostic biomarkers and targets for the treatment of patients with the disease.

\section{Materials and methods}

Animal models. A total of 20 male C57BL/6 mice (6-8 weeks old, weighing 17-20 g) were obtained from JSJ Laboratory Animal Co., Ltd. (Shanghai, China). The experimental protocols involving the animals were approved by the Institutional Animal Care and Use Committee of Huadong Hospital, Fudan University (Shanghai, China). All mice were housed in room temperature and relative humidity of $\sim 60 \%$ environment with a 12-h light-dark cycle, and fed with normal chow and water. Following acclimatization for 1 week, mice were randomly assigned into two groups, including the hypoxia-PH group $(n=10)$ and the control group $(n=10)$. The hypoxia-PH mice were exposed to hypoxia $\left(10 \% \mathrm{O}_{2}\right)$ in a normobaric chamber for 21 days (17). The control mice were housed in room air. The feeding conditions were the same for both groups.

PH measurements. The right ventricular systolic pressure (RVSP) and right ventricular hypertrophy (RVH) were considered to be indicators of $\mathrm{PH}$. At the end of the treatment period, mice were anesthetized with $20 \%$ urethane $(0.4 \mathrm{ml} / 100 \mathrm{~g}$, intraperitoneal), and then a catheter was inserted into the right ventricle (RV) via the right jugular vein. RVSP was measured by a gauge pressure transducer (RM-6000; Nihon Kohden Corporation, Tokyo, Japan). Animals were then sacrificed and the hearts were collected. The RV was dissected from the left ventricle (LV) and septum, and the two ventricles were then weighed. The RVH was determined based on the ratio of the RV weight compared with the weight of the LV and septum [calculated as $\mathrm{RV} /(\mathrm{LV}+\mathrm{S})$ ]. Additionally, lung tissues were harvested and stored at $-80^{\circ} \mathrm{C}$ for subsequent circRNA microarray analysis.

CircRNA microarray analysis. Lung samples from the hypoxia-PH group were randomly distributed into three subgroups for further analysis. The two subgroups had three lung samples from the hypoxia-PH group and the other subgroup has four lung samples from the hypoxia-PH group. The same grouping strategy was applied to the control group. Total RNA from each subgroup was isolated using TRIzol reagent (Invitrogen; Thermo Fisher Scientific, Inc., Waltham, MA, USA) according to the manufacturer's protocol. The RNA concentration was quantified using a NanoDrop spectrophotometer (ND-1000; Thermo Fisher Scientific, Inc., Wilmington, DE, USA), while RNA integrity was determined using gel electrophoresis. Following RNA quality control, fluorescence labeling and microarray hybridization were performed according to the manufacturer's protocol for the Arraystar Super RNA Labeling kit (Arraystar, Inc., Rockville, MD, USA). Briefly, linear RNAs were eliminated using RNase R (Epicentre; Illumina, Inc., San Diego, CA, USA), and the enriched circRNAs were subsequently amplified and transcribed into fluorescent complementary RNA (cRNA) by employing a random priming method (Arraystar Super RNA Labeling kit; Arraystar, Inc.). Labeled cRNA was then purified using the RNeasy Mini kit (Qiagen GmbH, Hilden, Germany). Labeled cRNA quality and quantity were determined using the NanoDrop ND-1000 spectrophotometer. Subsequently, the labeled cRNA was fragmented and hybridized onto an Arraystar mouse circRNA microarray (6x7K; Arraystar, Inc.). Subsequent to washing with gene expression wash buffer 1 (Agilent Technologies, Inc., Santa Clara, CA, USA) for $1 \mathrm{~min}$ at $37^{\circ} \mathrm{C}$ and then gene expression wash buffer 2 (Agilent Technologies, Inc.) for $1 \mathrm{~min}$ at $37^{\circ} \mathrm{C}$, the hybridized arrays were scanned using an Axon GenePix 4000B microarray scanner (Molecular Devices, LLC, Sunnyvale, CA, USA).

CircRNA data analysis. GenePix Pro 6.0 software (Axon; Molecular Devices, LLC) was used to analyze the scanned images of circRNA microarray in order to extract the raw data. Normalization and data processing were accomplished using the R software package (version 3.4.1; R Foundation Inc., Vienna, Austria). The statistical significance of the expression of circRNAs between the two groups was estimated by fold-change filtering and an unpaired t-test. To increase the number of circRNAs detected, the screening criterion for differentially expressed circRNAs was a fold-change of $\geq 1.5$ and a P-value of $<0.05$. Hierarchical cluster analyses, box plot, volcano plot and scatter plot were used to display the differential expression of circRNAs between the two groups.

$R T-q P C R$ validation. The top 12 candidate dysregulated circRNAs (including six upregulated circRNAs and six downregulated circRNAs) were selected for validation by RT-qPCR. Briefly, total RNA was extracted from the two 
Table I. Primers of validated circRNAs.

\begin{tabular}{lll}
\hline Name & \multicolumn{1}{c}{ Forward } & \multicolumn{1}{c}{ Reverse } \\
\hline GAPDH & 5'-GTTGTCTCCTGCGACTTCA-3' & 5'-GCCCCTCCTGTTATTATGG-3' \\
mmu_circRNA_010744 & 5'-GGCTTACACCTCAACTACCATCC-3' & 5'-CCCAGCATTCCGAAGAACC-3' \\
mmu_circRNA_014597 & 5'-CTTCAATGATTTTCACCTCCAG-3' & 5'-AGCGGTCATCCATGCTTATAT-3' \\
mmu_circRNA_008379 & 5'-CTGTCCCAACTGTAAAGAAGGTG-3' & 5'-CATCGGTTTGGTGCTCCTC-3' \\
mmu_circRNA_000267 & 5'-GGCACCCAACCTCATCTTC-3' & 5'-TTCGCACTTCTCATAGTCACTCA-3' \\
mmu_circRNA_015666 & 5'-ACGCCCAGGATTATGAAGAGG-3' & 5'-GGTACTGTGGTGAGTCTGGACGA-3' \\
mmu_circRNA_013176 & 5'-CCCCACTGAACAGGAAGAAGA-3' & 5'-ACGATACAAGCACCCAGATAGG-3' \\
mmu_circRNA_016128 & 5'-CTTCTGGTTCCTGGAGTTGG-3' & 5'-AGTTCTGGGATGCTTGCTTTA-3' \\
mmu_circRNA_018351 & 5'-GCTCAACCTCATCCAGCACG-3' & 5'-GCGAGTCACAGCCTTCCAT-3' \\
mmu_circRNA_004592 & 5'-TGTGGTTGGCAAGGATGTC-3' & 5'-CGCATGGGAACTGTAGTAAGAT-3' \\
mmu_circRNA_018701 & 5'-GAAGATAGTGCCTTTCAGCCATAC-3' & 5'-CACTCCCGTTTATCCCACTCA-3' \\
mmu_circRNA_016636 & 5'-GGAAAGGAGCAAACACGAAGG-3' & 5'-TGTCAGCGGCAAGTCATCG-3' \\
mmu_circRNA_010120 & 5'-GCAGTACGCCGTCATCAGTG-3' & 5'-GGAAGGCAGCAGACAAGAGC-3'
\end{tabular}

circRNA, circular RNA.

groups using TRIzol reagent (Thermo Fisher Scientific, Inc.). The concentration of total RNA was measured by a NanoVue Plus spectrophotometer (GE Healthcare, Inc., Chicago, IN, USA) and total RNA ( $1 \mu \mathrm{g})$ was reverse transcribed into cDNA using a PrimeScript ${ }^{\mathrm{TM}}$ RT Reagent kit (Takara Bio, Inc., Otsu, Japan) according to the manufacturer's protocol. The RT reaction was conducted at $37^{\circ} \mathrm{C}$ for $15 \mathrm{~min}$ and $85^{\circ} \mathrm{C}$ for $5 \mathrm{sec}$. Next, the RT-qPCR reaction was performed using $\mathrm{SYBR}^{\circledR}$ Premix Ex Taq $^{\mathrm{TM}}$ (Takara Bio, Inc.) using a Bio-Rad Real-Time PCR system (Bio-Rad Laboratories, Inc., Hercules, CA, USA). The amplification reaction involved initial denaturation at $95^{\circ} \mathrm{C}$ for $1 \mathrm{~min}$, and 40 cycles of denaturation at $95^{\circ} \mathrm{C}$ for $5 \mathrm{sec}$ and $60^{\circ} \mathrm{C}$ for $30 \mathrm{sec}$. The primers for the circRNAs were designed in divergent orientation, and the primer sequence pairs are presented in Table I. The relative level of circRNA expression for each target gene is shown as the $2^{-\Delta \Delta C Q}$ value (18).

Bioinformatics analysis. CircRNAs contain miRNA response elements (MREs) and function as miRNA sponges, which regulate miRNA and downstream gene expression through a competing endogenous RNA (ceRNA) mechanism (19). Putative circRNA-miRNA-mRNA networks were generated using various bioinformatics tools. The circRNA-miRNA interaction was predicted using the miRNA target prediction software (Arraystar, Inc.), which is based on the TargetScan (20) and miRanda (21). The top five circRNA-bound miRNAs were identified based on the prediction of miRNA binding sites. To ensure the efficiency and accuracy of the analysis, circRNAs were selected (fold-change $\geq 2$ and $\mathrm{P}<0.05$ ) and their target genes were predicted using miRWalk 2.0, which integrates miRWalk, RNA22, miRanda and TargetScan (22). The top 10 circRNA target genes for each miRNA were identified and used to construct circRNA-miRNA-mRNA networks using Cytoscape software (version 3.5.1; The Cytoscape Consortium, San Diego, CA, USA). The functional roles of the circRNA-target genes were further analyzed using Gene Ontology (GO; geneontology.org) and were classified into three categories, including biological process (BP), molecular function (MF) and cellular component (CC). In addition, the biological pathways of circRNA-target genes were identified using Kyoto Encyclopedia of Genes and Genomes (KEGG; www.kegg.jp). The GO and KEGG analyses were performed using DIANA-miRPath, version 3.0 (23).

Statistical analysis. Validated data from RT-qPCR are expressed as the mean \pm standard error of the mean. Differences between the groups were assessed by SPSS statistical software version 19.0 (IBM Corp., Armonk, NY, USA) using one-way analysis of variance. $\mathrm{P}<0.05$ was considered to indicate a statistically significant difference.

\section{Results}

Expression profile of circRNAs. The murine model of hypoxia-induced $\mathrm{PH}$ was evaluated by measuring the RVSP and RVH (24). When compared with the control group, the hypoxia-induced $\mathrm{PH}$ group had significantly increased levels of RVSP (30.77 vs. $22.96 \mathrm{mmHg}, \mathrm{P}<0.01)$ and RVH (0.39 vs. 0.28, $\mathrm{P}<0.01$; Fig. 1A and B). Subsequent to obtaining the aforementioned measurements, the lungs were harvested for circRNA microarray detection. The box plot in Fig. 2A demonstrates that there was no abnormal distribution of circRNA expression in the six samples after normalization. Next, a scatter plot was used to assess the expression variation of circRNAs between the hypoxia-PH and control groups (Fig. 2B). The hierarchical cluster analysis revealed the differential expression profile of circRNAs in these two groups (Fig. 2C). A volcano plot was then constructed using fold-change values and P-values in order to visualize the differential expression of circRNAs between the two groups (Fig. 2D). In total, 64 differentially expressed circRNAs were identified between the hypoxia-PH and control groups, of which 23 circRNAs were upregulated and 41 circRNAs were downregulated. The top 20 upregulated and top 20 downregulated circRNAs are listed in Table II. 

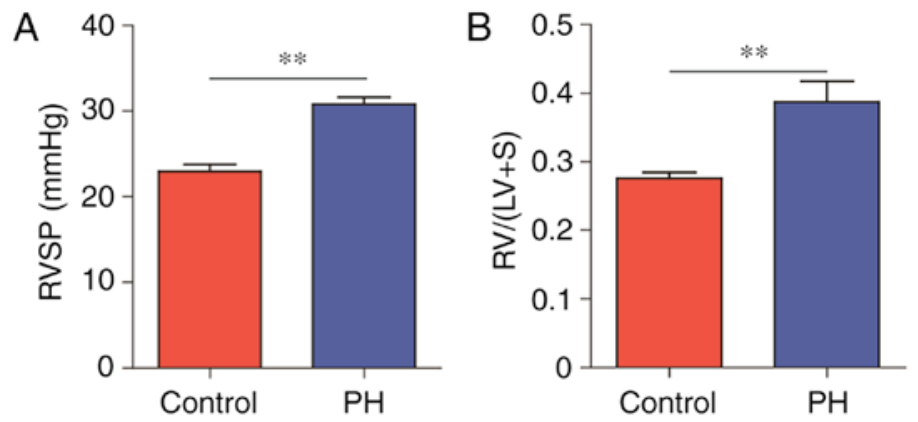

Figure 1. Measurement of RVSP and RVH in mice with hypoxia-induced pulmonary hypertension. (A) RVSP was significantly increased in mice with hypoxia-induced PH, compared with that in the control group. (B) RVH was significantly increased in mice with hypoxia-induced PH, compared with that in the control group. Values are presented as the mean \pm standard error of the mean $(n=10) .{ }^{* *} \mathrm{P}<0.01$. RVSP, right ventricular systolic pressure; RVH, right ventricular hypertrophy; $\mathrm{PH}$, pulmonary hypertension; $\mathrm{RV} /(\mathrm{LV}+\mathrm{S})$, right ventricle/(left ventricle + septum) weight.
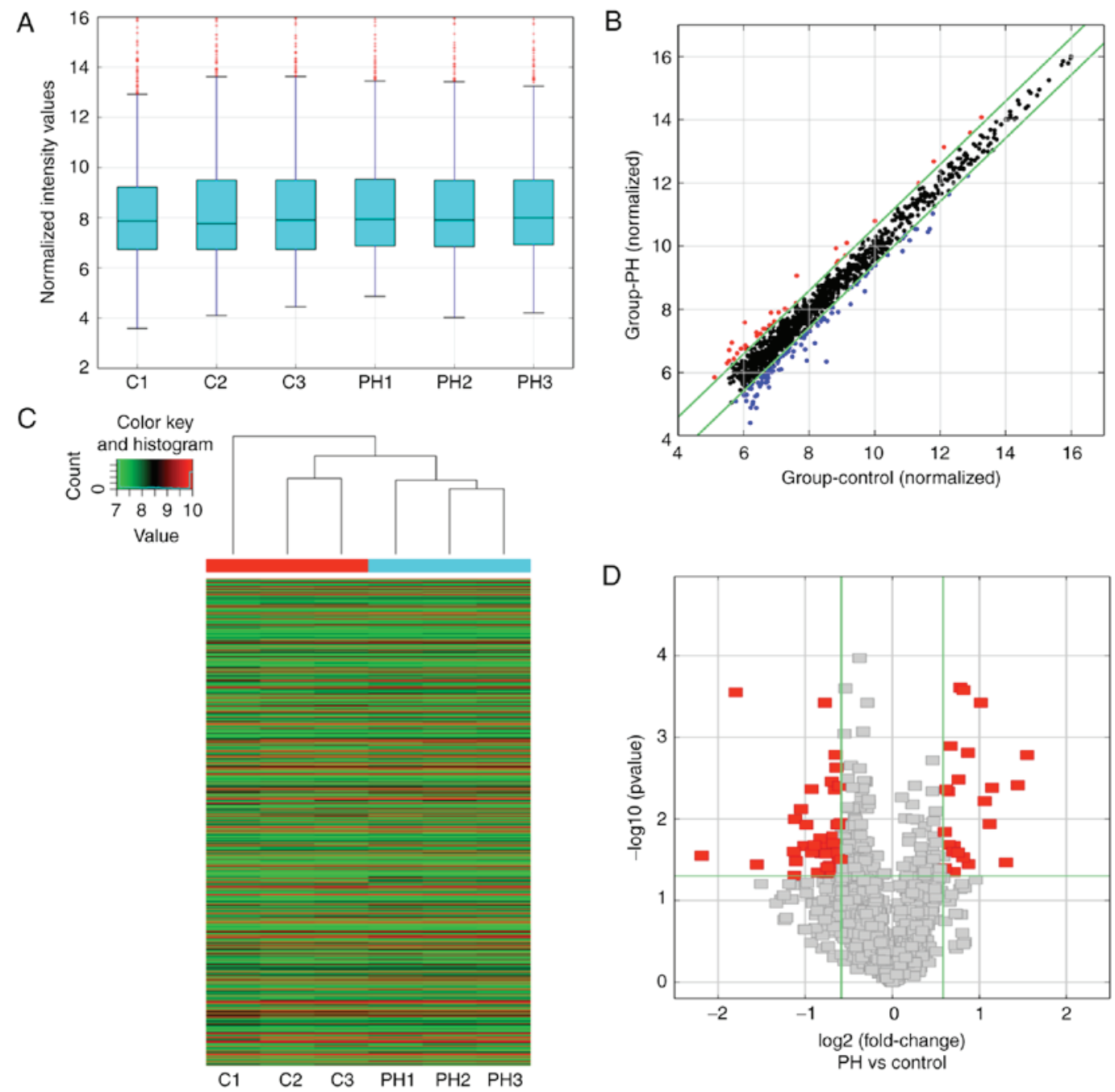

Figure 2. Microarray analysis of differential expression of circRNAs in mice with hypoxia-induced PH. (A) Box plot showing that the normalized intensity of the hypoxia-PH and control groups was nearly the same. (B) Scatter plot demonstrating the expression variation of the circRNAs in the two groups. The values of $\mathrm{x}$ and $\mathrm{y}$-axes in the scatterplot represent the normalized signal values of the samples ( $\log 2$ scaled). The red points outside the top green line represent the upregulated circRNAs with $\geq 1.5$-fold change and the blue points outside bottom green line represent the down-regulated circRNAs with $\geq 1.5$-fold change. (C) Hierarchical cluster analysis revealed the expression profile of the dysregulated circRNAs in the two groups. (D) Volcano plot showing the differentially expressed circRNAs in the two groups, with red points representing the significantly different circRNAs. PH, pulmonary hypertension; circRNA, circular RNA.

Validation of circRNAs expression. In order to validate the results of the circRNA microarray, 12 differentially expressed circRNAs (including six upregulated and six downregulated circRNAs) were selected for RT-qPCR analysis on the basis of the fold-change and P-values. The six upregulated circRNAs were as follows: mmu_circRNA_004592, mmu_circRNA_018701, mmu_circRNA_010744, mmu_circRNA_015666, mmu_circRNA_016128 
Table II. Top 20 upregulated and top 20 downregulated circRNAs in mice with hypoxia-induced pulmonary hypertension.

\begin{tabular}{|c|c|c|c|c|c|c|}
\hline CircRNA & Fold change & P-value & Regulation & CircRNA type & Chromosome & Gene symbol \\
\hline mmu_circRNA_004592 & 2.925894 & 0.001652 & Up & Exonic & $\mathrm{X}$ & Fam120c \\
\hline mmu_circRNA_018701 & 2.717783 & 0.003849 & Up & Exonic & 10 & Jmjd1c \\
\hline mmu_circRNA_010744 & 2.469385 & 0.03412 & $\mathrm{Up}$ & Exonic & 1 & Sntg1 \\
\hline mmu_circRNA_015666 & 2.211677 & 0.004139 & Up & Exonic & 4 & Ikbkap \\
\hline mmu_circRNA_016128 & 2.168588 & 0.011483 & Up & Exonic & 7 & Nars2 \\
\hline mmu_circRNA_016636 & 2.085027 & 0.006036 & Up & Exonic & 12 & Ttc7b \\
\hline mmu_circRNA_002378 & 2.02187 & 0.000377 & Up & Intronic & 2 & Kif5c \\
\hline mmu_circRNA_011174 & 1.834095 & 0.035723 & $\mathrm{Up}$ & Intragenic & 17 & XLOC_010800 \\
\hline mmu_circRNA_004941 & 1.82896 & 0.001544 & $\mathrm{Up}$ & Intragenic & 13 & Cdc14b \\
\hline mmu_circRNA_015350 & 1.759724 & 0.000263 & Up & Exonic & 1 & Ncoa2 \\
\hline mmu_circRNA_011414 & 1.756762 & 0.029613 & Up & Exonic & 18 & Tcf4 \\
\hline mmu_circRNA_008988 & 1.717354 & 0.000246 & Up & Exonic & 4 & Unc13b \\
\hline mmu_circRNA_011332 & 1.691847 & 0.003271 & Up & Intragenic & 8 & Large \\
\hline mmu_circRNA_014444 & 1.684291 & 0.025843 & Up & Exonic & 4 & Haus6 \\
\hline mmu_circRNA_006839 & 1.632387 & 0.044651 & Up & Intronic & 17 & Crim1 \\
\hline mmu_circRNA_007240 & 1.632184 & 0.021443 & Up & Exonic & 5 & Bre \\
\hline mmu_circRNA_017590 & 1.627719 & 0.025193 & Up & Exonic & 6 & Ccdc132 \\
\hline mmu_circRNA_013053 & 1.59081 & 0.001276 & Up & Exonic & 6 & Gmcl1 \\
\hline mmu_circRNA_000113 & 1.566056 & 0.02078 & Up & Antisense & 12 & Rian \\
\hline mmu_circRNA_012851 & 1.561305 & 0.004609 & $\mathrm{Up}$ & Exonic & 2 & Arhgap21 \\
\hline mmu_circRNA_010120 & 4.557204 & 0.028091 & Down & Exonic & 14 & Cryl1 \\
\hline mmu_circRNA_018351 & 3.479684 & 0.00028 & Down & Exonic & 8 & Zfhx3 \\
\hline mmu_circRNA_013176 & 2.940519 & 0.036055 & Down & Exonic & 4 & Frmd3 \\
\hline mmu_circRNA_012978 & 2.189961 & 0.025108 & Down & Intronic & 17 & Tsc2 \\
\hline mmu_circRNA_007835 & 2.181447 & 0.049532 & Down & Intragenic & 13 & Cdc14b \\
\hline mmu_circRNA_008379 & 2.167655 & 0.010006 & Down & Exonic & 2 & Sp3 \\
\hline mmu_circRNA_001170 & 2.150695 & 0.032906 & Down & Intragenic & 10 & Hsp90b1 \\
\hline mmu_circRNA_000267 & 2.068873 & 0.007548 & Down & Exonic & 3 & Plekho1 \\
\hline mmu_circRNA_014597 & 2.014597 & 0.021489 & Down & Exonic & 1 & Kifap3 \\
\hline mmu_circRNA_003780 & 1.983159 & 0.011809 & Down & Exonic & 13 & Cdyl \\
\hline mmu_circRNA_005086 & 1.901804 & 0.004295 & Down & Intragenic & 10 & Tbpl1 \\
\hline mmu_circRNA_010464 & 1.894022 & 0.025737 & Down & Exonic & 6 & Ezh2 \\
\hline mmu_circRNA_009705 & 1.859579 & 0.020918 & Down & Exonic & 7 & Mettl9 \\
\hline mmu_circRNA_001362 & 1.809006 & 0.045429 & Down & Exonic & 5 & Gm15800 \\
\hline mmu_circRNA_018468 & 1.776092 & 0.017304 & Down & Exonic & 18 & Arhgap26 \\
\hline mmu_circRNA_006562 & 1.752095 & 0.026256 & Down & Intragenic & 10 & Erbb3 \\
\hline mmu_circRNA_002959 & 1.727222 & 0.020858 & Down & Exonic & 8 & Phkb \\
\hline mmu_circRNA_005155 & 1.710652 & 0.000376 & Down & Exonic & 16 & Robo1 \\
\hline mmu_circRNA_000270 & 1.694113 & 0.026716 & Down & Exonic & 2 & Fam188a \\
\hline mmu_circRNA_009674 & 1.687043 & 0.046010 & Down & Exonic & 5 & Rnf4 \\
\hline
\end{tabular}

circRNA, circular RNA.

and mmu_circRNA_016636. The six downregulated circRNAs were as follows: mmu_circRNA_010120, mmu_circRNA_018351, mmu_circRNA_013176, mmu_circRNA_008379, mmu_circRNA_000267 and mmu_circRNA_014597. Analysis by RT-qPCR revealed that five of the upregulated circRNAs and two of the downregulated circRNAs, exhibited changes in their expression levels that were consistent with the results of the circRNA microarray. More specifically, mmu_circRNA_004592, mmu_circRNA_018701, mmu_circRNA_010744, mmu_ circRNA_015666 and mmu_circRNA_016636 were found to be significantly upregulated in the hypoxia-PH group compared with their levels in the control group (Fig. 3A-E), while mmu_circRNA_018351 and mmu_circRNA_008379 
A

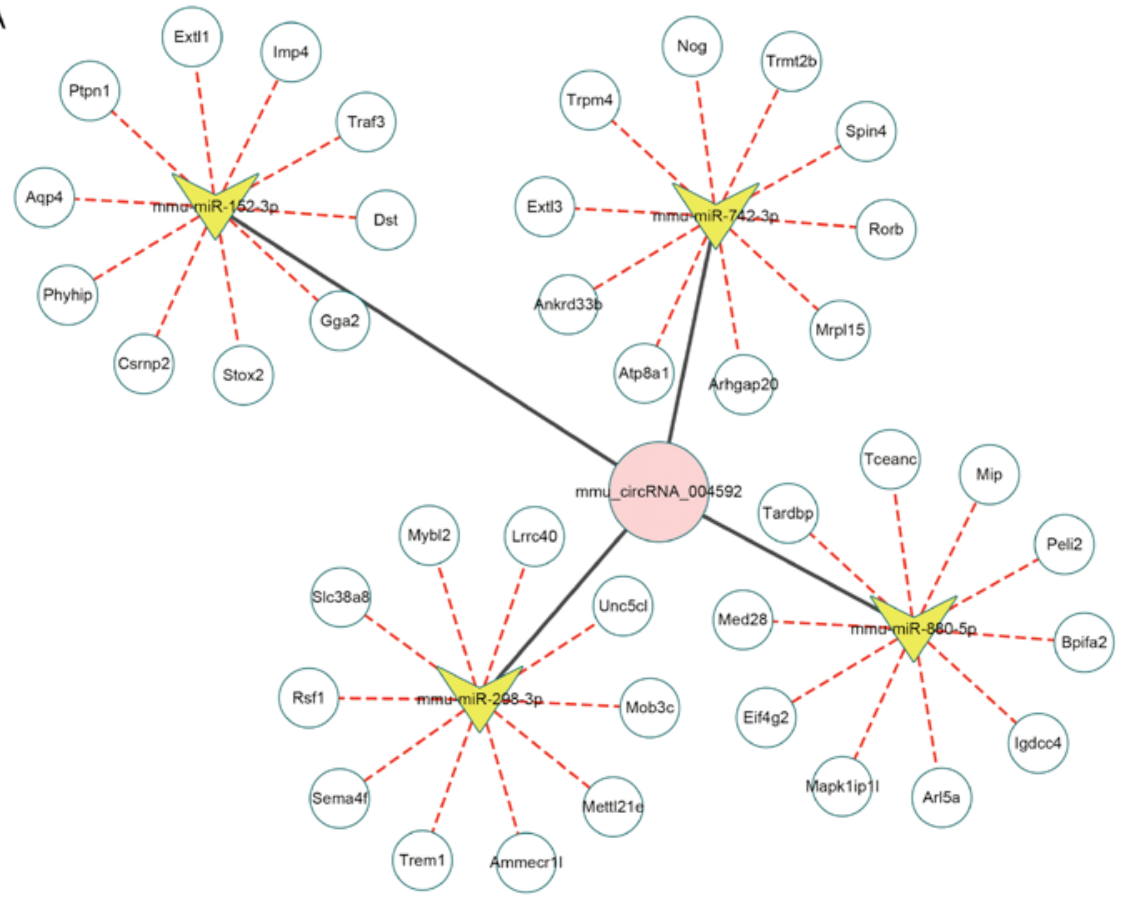

$\mathrm{B}$
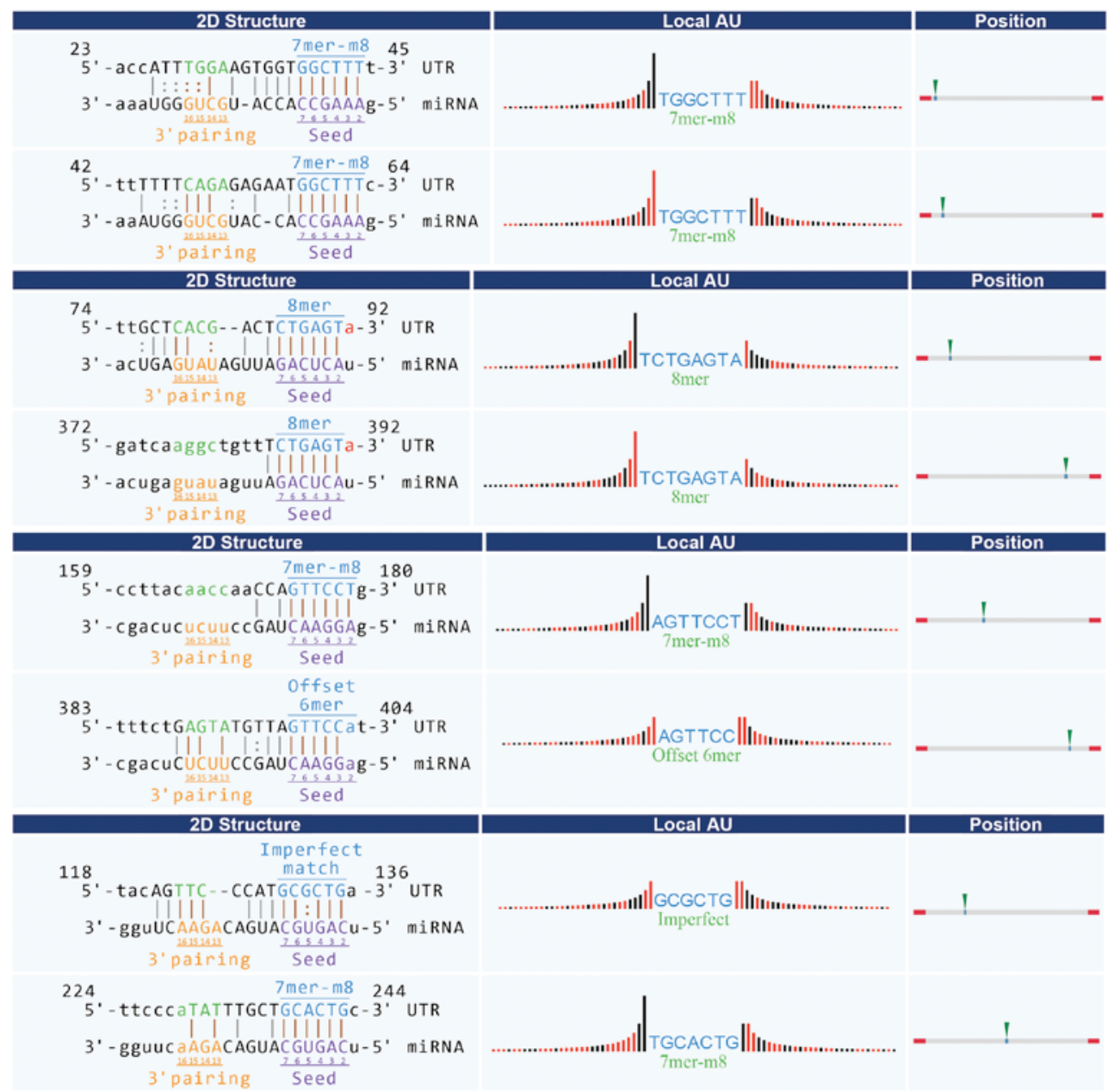

Figure 4.CircRNA-miRNA-mRNA network of mmu_circRNA_004592.(A) Putative circRNA-miRNA-mRNA network of mmu_circRNA_004592 (pink circle) was constructed using bioinformatics tools. (B) Predicted miRNA response elements for mmu_circRNA_004592. circRNA, circular RNA; miRNA, microRNAs.

analysis shown in Fig. 4A. For mmu_circRNA_004592, the circRNA-miRNA-mRNA network was constructed (Fig. 4A).
The upregulated mmu_circRNA_004592 was predicted to inhibit the expression level of mmu-miR-742-3p, 

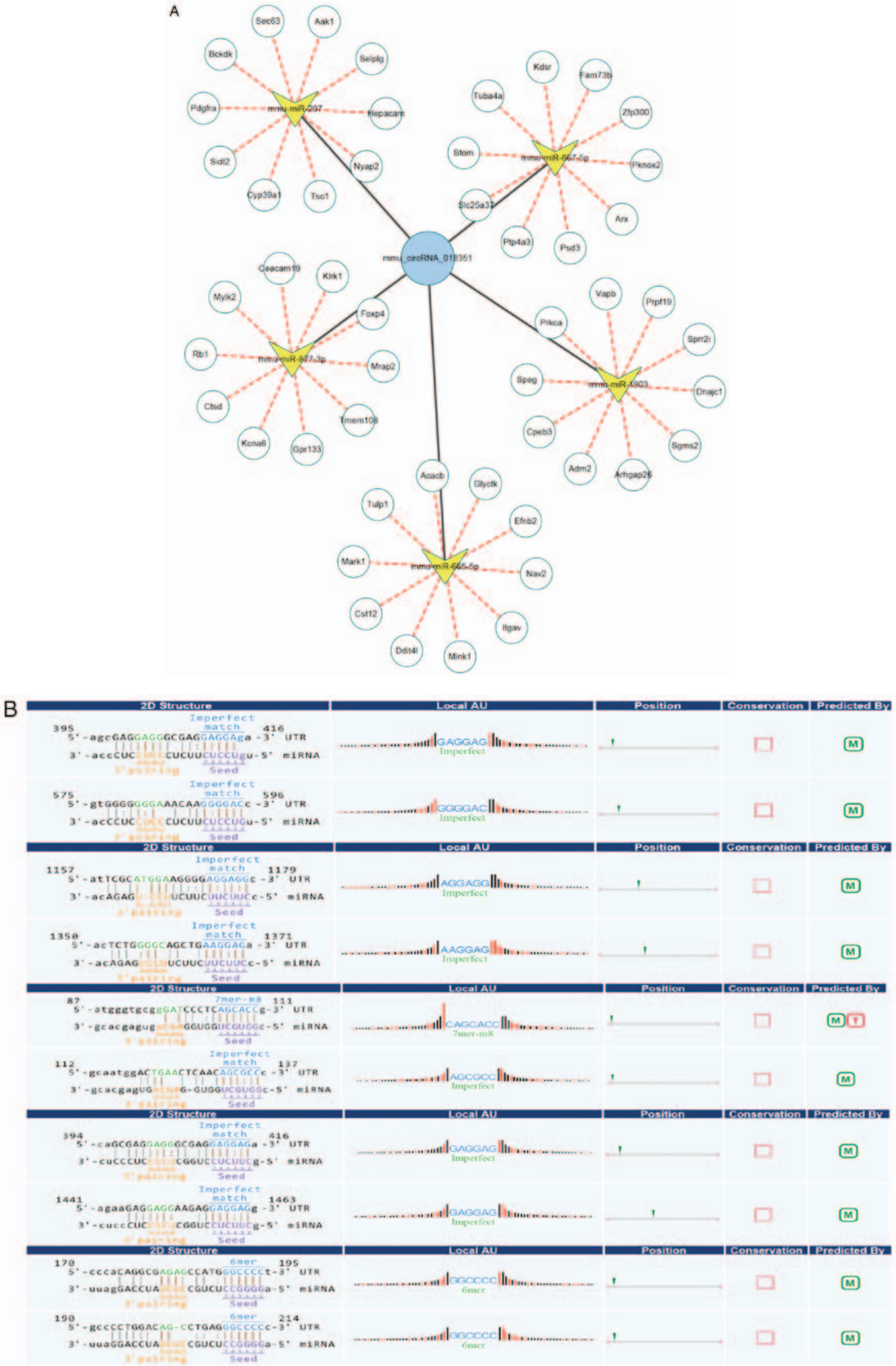

Figure 5.CircRNA-miRNA-mRNA network of mmu_circRNA_018351. (A) Putative circRNA-miRNA-mRNA network of mmu_circRNA_018351 (blue circle) was constructed using bioinformatics tools. (B) Predicted miRNA response elements for mmu_circRNA_018351.circRNA, circular RNA; miRNA, microRNAs.

mmu-miR-880-5p, mmu-miR-298-3p and mmu-miR-152-3p, The top ten miRNA-targeted genes are presented in Fig. 4A. and further promote the expression of miRNA-targeted genes. The sequence analysis of MREs of mmu_circRNA_004592 
A

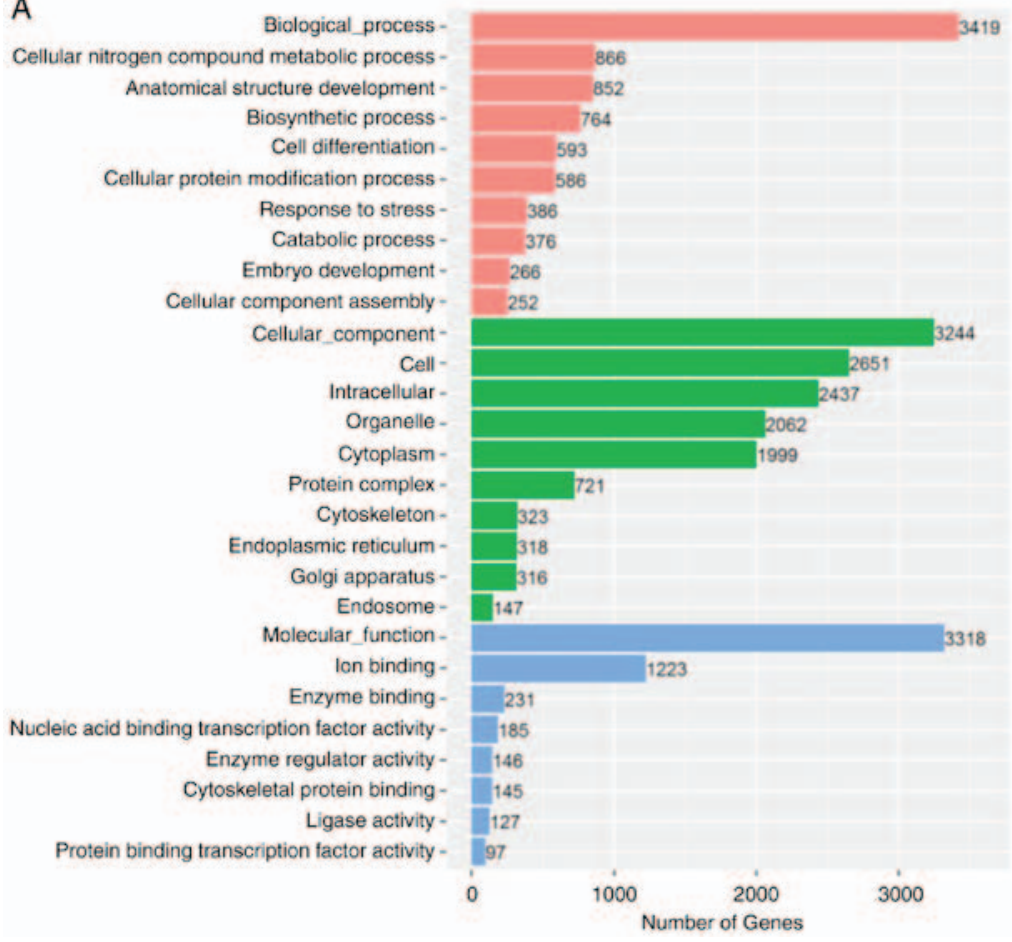

B

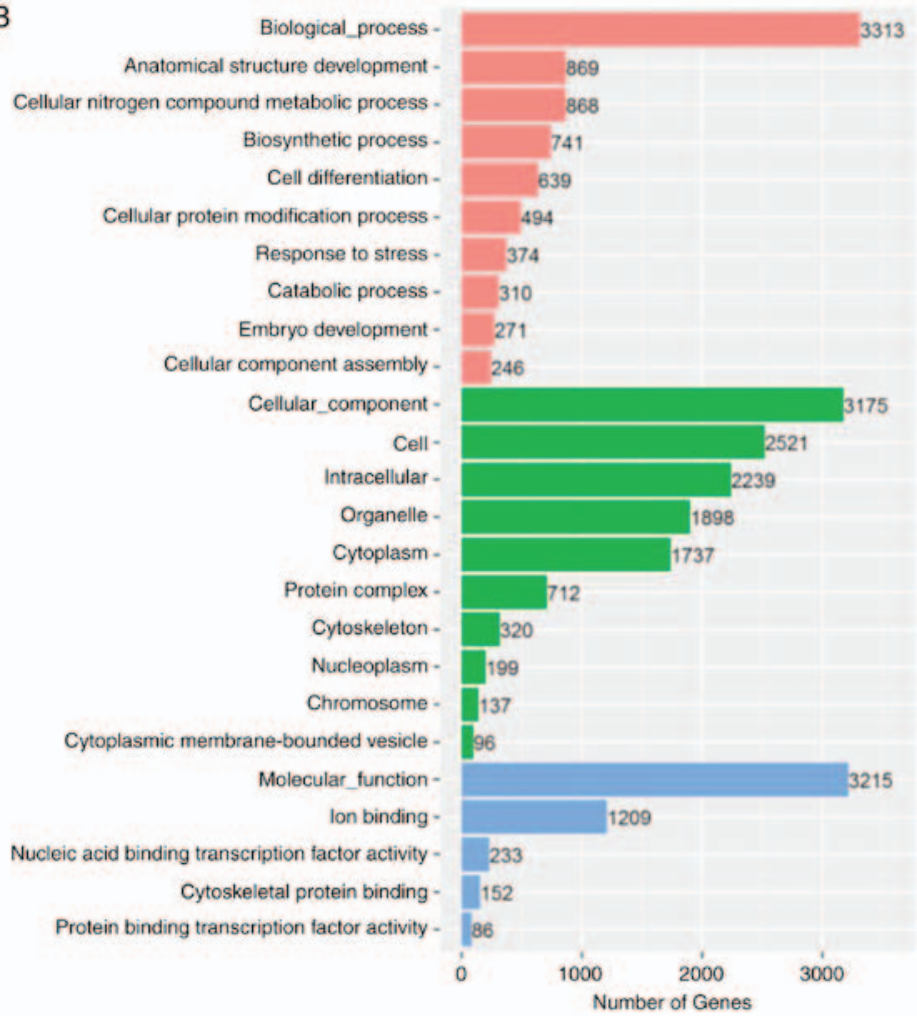

Biological_process

Cellular_component

Molecular_function
Biological_process

Cellular_component

Molecular function

Figure 6. GO analysis of the target genes of circRNAs. The target genes of dysregulated circRNAs with 2-fold change and P $<0.05$ were identified using bioinformatics tools. Functions of the target genes of the (A) upregulated and (B) circRNAs were enriched by GO analysis. The total number of genes is shown next to each bar. GO, Gene Ontology; circRNA, circular RNA.

was presented in Fig. 4B. The 2D structure demonstrated the MRE sequence, the target miRNA seed type and the 3 pairing sequence for mmu_circRNA_004592. Similarly, the circRNA-miRNA-mRNA network for mmu_circRNA_018351 was constructed (Fig. 5A). The downregulated mmu circRNA_018351 was predicted to increase the expression level of mmu-miR-6992-3p, mmu-miR-5133, mmu-miR-6936-5p, mmu-miR-6938-5p and mmu-miR-7015-3p, and further reduce the expression of miRNA-targeted genes. The top ten miRNA-targeted genes are presented in Fig. 5A. The sequence analysis of MREs of mmu_circRNA_018351 identified the MRE sequence, the target miRNA seed type and 
A

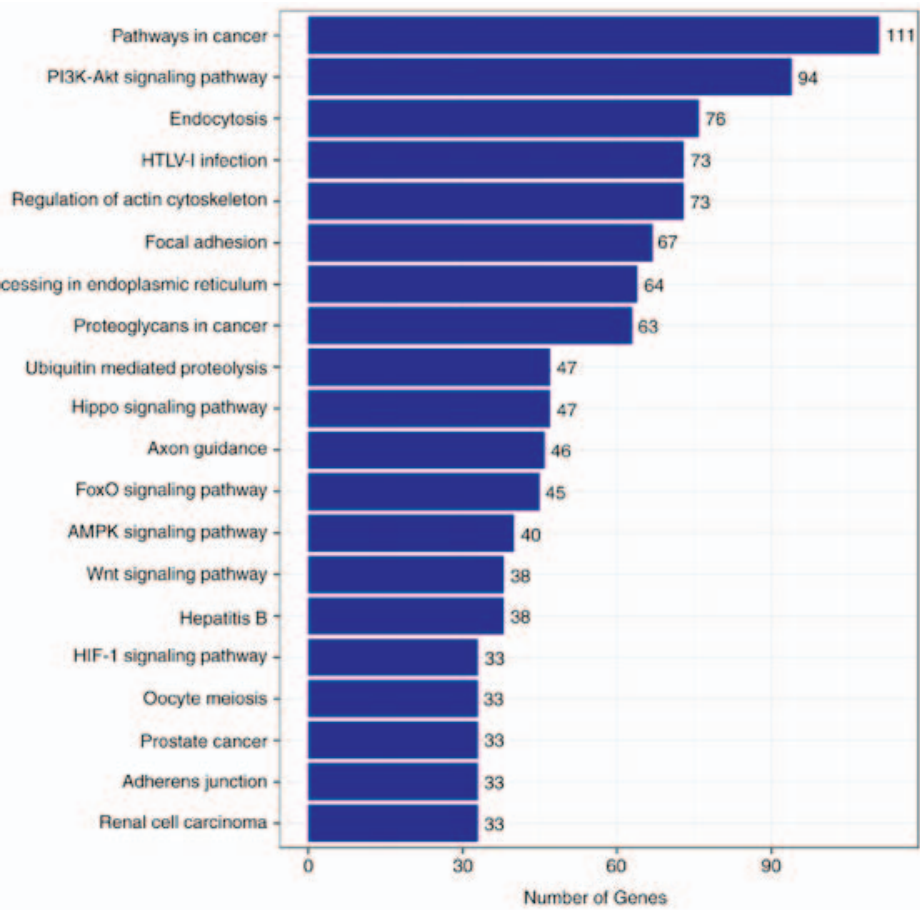

B

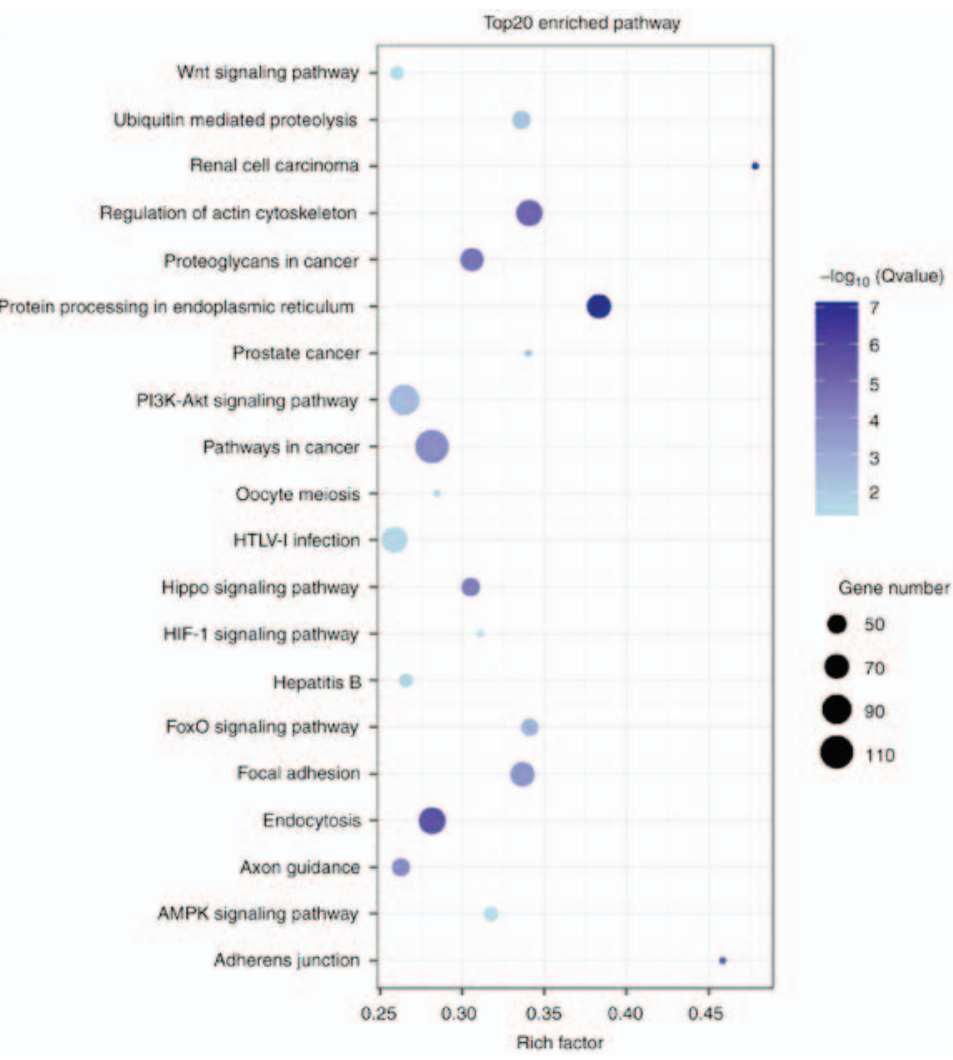

Figure 7. KEGG pathway analysis of the target genes of circRNAs. circRNAs with 2 -fold change and $\mathrm{P}<0.05$ were selected from the dysregulated circRNAs, and the target genes of these circRNAs were identified using bioinformatics tools. (A) The top 20 pathways of the target genes of upregulated circRNAs were identified using KEGG analysis according to the number of enriched genes. (B) The top 20 pathways of the target genes of upregulated circRNAs were identified using KEGG analysis according to the rich factor, Q value and the number of enriched genes.

the 3' pairing sequence for mmu_circRNA_004592 in 2D sequence (Fig. 5B).

GO and KEGG analyses for circRNA-targeting genes. CircRNAs with a fold-change of $\geq 2$ and a P-value of $<0.05$ were selected from the dysregulated circRNAs in the hypoxia-induced PH group, and the role of these circRNAs was further investigated by GO and KEGG analyses. For the target genes of the upregulated circRNAs, the top 10 enriched GO terms in the BP and CC categories, as well as the total eight GO terms in the MF category were identified. The top three enriched GO terms were biological processes, cellular nitrogen compound metabolic processes and anatomical structure development in BP; cellular component, cell and 

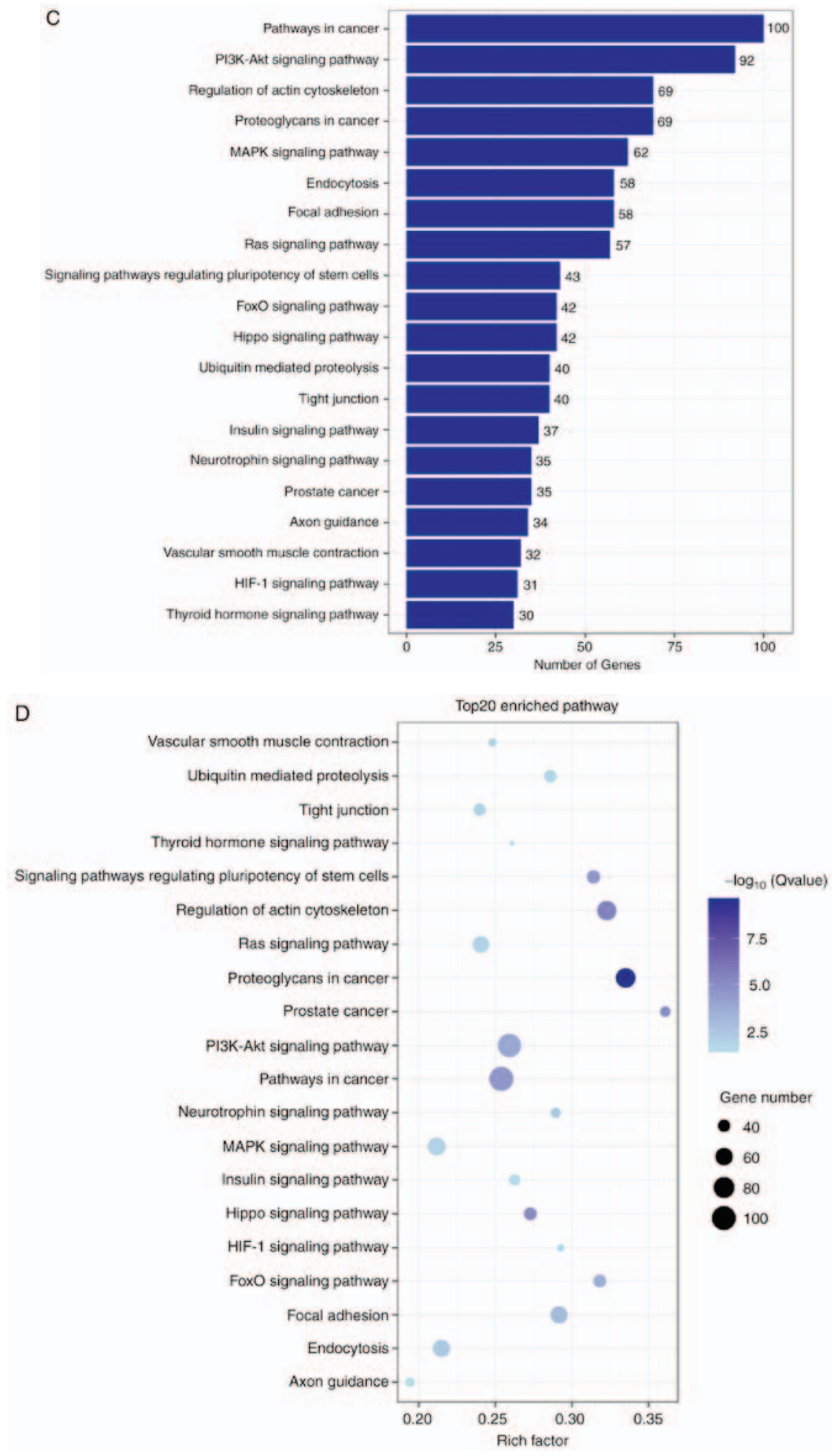

Figure 7. Continued. (C) The top 20 pathways of the target genes of downregulated circRNAs were identified using KEGG analysis according to the number of enriched genes. (D) The top 20 pathways of the target genes of downregulated circRNAs were identified using KEGG analysis according to rich factor, $Q$ value and the number of enriched genes. KEGG, Kyoto Encyclopedia of Genes and Genomes; circRNA, circular RNA.

intracellular in $\mathrm{CC}$; molecular function, ion binding and enzyme binding in MF (Fig. 6A). For the target genes of the downregulated circRNAs, the top 10 enriched GO terms in the $\mathrm{BP}$ and $\mathrm{CC}$ categories, as well as the total five GO terms in the MF category were displayed. The top three enriched were biological processes, anatomical structure development and cellular nitrogen compound metabolic process in BP; cellular component, cell and intracellular in $\mathrm{CC}$; molecular function, ion binding and nucleic acid binding transcription factor activity in MF (Fig. 6B).

The top 20 KEGG pathways were also identified for the target genes of the upregulated and downregulated circRNAs, respectively (Fig. 7A-D). The top five enriched pathways for the target genes of the upregulated circRNAs included pathways in cancer, the PI3K-Akt signaling pathway, endocytosis, HTLV-I infection and regulation of actin cytoskeleton (Fig. 7A and B). 
Furthermore, the top five enriched pathways for the target genes of the downregulated circRNAs involved pathways in cancer, the PI3K-Akt signaling pathway, regulation of actin cytoskeleton, proteoglycans in cancer, and the MAPK signaling pathway (Fig. 7C and D).

\section{Discussion}

To the best of our knowledge, the present study is the first to perform circRNA microarray analysis to detect the differential expression profile of circRNAs in the lungs of mice with hypoxia-induced $\mathrm{PH}$. In total, 23 significantly upregulated and 41 significantly downregulated circRNAs were identified. Of these, 12 differentially expressed circRNAs were selected for further validation using RT-qPCR. Bioinformatics tools were used to construct the circRNA-miRNA-mRNA networks, and the putative functions and pathways were obtained using GO and KEGG enrichment analyses. The results from circRNA-miRNA-mRNA networks analysis and GO and KEGG analysis suggested that dysregulated circRNAs may serve key roles in the pathogenesis of hypoxia-induced $\mathrm{PH}$.

Pulmonary vascular remodeling is a key feature of hypoxia-induced $\mathrm{PH}$ with associated dysfunction of endothelial cells, smooth muscle cells and fibroblasts (4). Recently, novel molecular mechanisms of $\mathrm{PH}$ have been identified with particular advances in 'omics' approaches (25). Several types of noncoding RNAs, such as long noncoding RNAs and miRNAs, participate in the pathological process of hypoxia-induced $\mathrm{PH}$, and certain of these are considered to be diagnostic or therapeutic targets $(26,27)$. CircRNAs are novel members of the non-coding RNA family and serve an important role in the development of several pulmonary diseases. The biological functions of circRNAs are varied; however, their function as miRNA sponges is likely to be the primary mechanism of action in a number of pulmonary diseases, including non-small cell lung cancer $(28,29)$, pulmonary tuberculosis (30), acute respiratory distress syndrome (31) and chronic thromboembolic PH (32). However, to the best of our knowledge, no previous study to date has investigated the expression of circRNAs in hypoxia-induced PH. Thus, to further elucidate the molecular RNA-based mechanism of PH, the circRNA expression profile in hypoxia-induced $\mathrm{PH}$ was determined in the present study, using microarray analysis and bioinformatics to identify several circRNAs that are potentially important in the development of hypoxia-induced $\mathrm{PH}$.

Based on the circRNA target prediction analysis, all of the differentially expressed circRNAs that were identified contained MREs to sponge different miRNAs. Of these, the two best candidate circRNAs were selected, namely mmu circRNA_004592 and mmu_circRNA_018351, and their circRNA-miRNA-mRNA networks were constructed using bioinformatics tools. The upregulated mmu_circRNA_004592 was predicted to negatively regulate the function of mmu-miR-742-3p, mmu-miR-6373, mmu-miR-880-5p, mmu-miR-298-3p and mmu-miR-152-3p. Although there is no direct evidence to show the role of these miRNAs in hypoxia-induced $\mathrm{PH}, \mathrm{Wu}$ et al (33) have previously reported that hypoxia significantly inhibited the expression of miR-152 in human umbilical vein endothelial cells and that miR-152 targeted ADAM17 to inhibit cell migration and proliferation.
Furthermore, several studies have suggested that miR-152 is a tumor suppressor (34). miR-152 has been reported to inhibit cancer cell proliferation, invasion and migration by targeting Wnt1 (35), PIK3CA (36), DNMT1 (37) and PTEN (38). Additionally, $\mathrm{Gu}$ et al (39) identified that miR-152 promoted senescence in stem cells from human dental pulp by targeting SIRT7 expression. Since mmu_circRNA_004592 expression was upregulated in the present study, it can be hypothesized that miR-152 is downregulated, which promotes proliferation, and inhibits senescence and apoptosis of endothelial cells, smooth muscle cells and fibroblasts in the pulmonary artery. These actions may further raise the pressure in the pulmonary vessels.

The current study also demonstrated that $\mathrm{mmu}_{-}$ circRNA_018351 was downregulated and had potential binding sites for mmu-miR-877-3p, mmu-miR-1903, mmu-miR-667-5p, mmu-miR-207 and mmu-miR-665-5p. Of these, miR-207 serves a protective role against cell apoptosis following ischemic stroke (40). In addition, miR-665 regulates insulin-like growth factor 2 to protect from sevoflurane anesthesia-induced cognitive dysfunction through inhibiting apoptosis (41). Based on the bioinformatics analysis of the present study, it is hypothesized that miR-207 and miR-665 are upregulated as a consequence of the downregulated mmu_circRNA_018351 expression to repress the apoptosis of endothelial cells, smooth muscle cells and fibroblasts in hypoxia-induced $\mathrm{PH}$.

Function and pathway analyses for the target genes of the circRNAs were performed to provide further insight into the mechanism of hypoxia-induced $\mathrm{PH}$. Among the enriched GO terms in BP, the cellular nitrogen compound metabolic process was identified. Several studies have demonstrated that the level of nitric oxide (NO) is decreased in patients with $\mathrm{PH}$ (42). $\mathrm{NO}$ is an important vasodilator for pulmonary vessels, and a decrease in the levels of NO could increase the vascular resistance and vascular pressure (43). Although current therapy targeting the NO pathway improves the clinical outcome of patients with $\mathrm{PH}$, the role of the NO pathway in the pathogenesis of pulmonary vascular remodeling in $\mathrm{PH}$ has not yet been fully elucidated (44). Thus, it is proposed that the circRNAs identified in the present study contribute to the pathophysiological effect of NO in PH and are potential clinical biomarkers.

Bioinformatics analysis also identified several hypoxia and vascular remodeling-associated pathways. The PI3K-Akt signaling pathway serves an important role in hypoxia-induced $\mathrm{PH}$ through promoting the proliferation of endothelial cells and smooth muscle cells $(45,46)$. Furthermore, the Hippo, Wnt, AMPK and HIF-1 signaling pathways, which were identified in KEGG pathway analysis in the present study, have been reported to have a close association with the development of hypoxia-induced $\mathrm{PH}(47,48)$. These findings suggest that circRNAs may be key modulators in the molecular mechanism of hypoxia-induced $\mathrm{PH}$ and that, in the future, specific circRNAs could be tested as diagnostic and therapeutic targets of hypoxia-induced $\mathrm{PH}$.

There are a number of limitations in the present study. Firstly, the differentially expressed circRNAs were identified only in a mouse model with hypoxia-induced $\mathrm{PH}$ and should be further validated in patients with PH. Secondly, the functions of the differentially expressed circRNAs were predicted using bioinformatics tools, and there were no 
further studies to demonstrate the role of candidate circRNAs in the pathological process of hypoxia-induced $\mathrm{PH}$. Thirdly, the circRNA-miRNA-mRNA networks were constructed in the current study according to the premise that the majority of circRNAs function as miRNA sponges. However, the molecular functions of $>99.9 \%$ of identified circRNAs remain unknown, and numerous other functions, such as translation and regulation of transcription and alternative splicing, have been reported (49). Thus, in the future, functional experiments should be conducted to elucidate the roles of these circRNAs in hypoxia-induced $\mathrm{PH}$.

In conclusion, the present study is the first to profile circRNA expression in hypoxia-induced PH. In total, 23 significantly upregulated and 41 significantly downregulated circRNAs were identified. The circRNA-miRNA interactions were also predicted and circRNA-miRNA-mRNA networks were constructed for two leading candidate circRNAs. Furthermore, GO and pathway analyses revealed the potential role of the target genes of circRNAs in hypoxia-induced $\mathrm{PH}$. The findings suggest that circRNAs serve a key role in the pathological process of hypoxia-induced $\mathrm{PH}$. Thus, candidate circRNAs are potential targets for treatment and potential biomarkers for diagnosis of patients with hypoxia-induced $\mathrm{PH}$.

\section{Acknowledgements}

Not applicable.

\section{Funding}

The study was supported by the State Key Basic Research Program Project (grant no. 2015CB553404), the National Natural Science Foundation of China (grant nos. 81770055, 81500026, 81570028 and 81600056), the Key Grant (grant nos. 81630001 and 81490533), the National Natural Science Foundation of China for Young Researcher (grant no. 81600056), the Shanghai Science and Technology Committee Grant (grant nos. 15DZ1930600, 15DZ193060 and 16ZR1405700) and the Shanghai Municipal Commission of Health and Family Planning (grant no. 201540370).

\section{Availability of data and materials}

All data generated or analyzed during this study are included in this published article.

\section{Authors' contributions}

SJX, HH and YLS designed and supervised the study. JW, MCZ and JZW made the mouse model. LLW and HYG contributed to data collection. CCC and XDT analyzed and interpreted the data. JW and MCZ drew the figures and wrote the manuscript. BK and YLS provided solutions for data analysis and edited the manuscript. All authors read and approved the final article.

\section{Ethics approval and consent to participate}

This study was approved by the Institutional Animal Care and Use Committee of Huadong Hospital, Fudan University, Shanghai, China.

\section{Patient consent for publication}

Not applicable.

\section{Competing interests}

The authors declare that they have no competing interests.

\section{References}

1. Thompson AAR and Lawrie A: Targeting vascular remodeling to treat pulmonary arterial hypertension. Trends Mol Med 23: 31-45, 2017

2. Task Force for Diagnosis and Treatment of Pulmonary Hypertension of European Society of Cardiology (ESC); European Respiratory Society (ERS); International Society of Heart and Lung Transplantation (ISHLT); Galiè N, Hoeper MM, Humbert M, Torbicki A, Vachiery JL, Barbera JA, Beghetti M, Corris P, Gaine S, Gibbs JS, et al: Guidelines for the diagnosis and treatment of pulmonary hypertension. Eur Heart J 32: 2493-2537, 2009

3. Naeije R and Dedobbeleer C: Pulmonary hypertension and the right ventricle in hypoxia. Exp Physiol 98: 1247-1256, 2013.

4. Ghofrani HA, Voswinckel R, Reichenberger F, Weissmann N, Schermuly RT, Seeger W and Grimminger F: Hypoxia- and non-hypoxia-related pulmonary hypertension-established and new therapies. Cardiovasc Res 72: 30-40, 2006.

5. Kylhammar D and Rådegran G: The principal pathways involved in the in vivo modulation of hypoxic pulmonary vasoconstriction, pulmonary arterial remodelling and pulmonary hypertension. Acta Physiol 219: 728-756, 2017.

6. McLaughlin VV: Looking to the future: A new decade of pulmonary arterial hypertension therapy. Eur Respir Rev 20: 262-269, 2011.

7. Liu L, Wang J, Khanabdali R, Kalionis B, Tai X and Xia S: Circular RNAs: Isolation, characterization and their potential role in diseases. RNA Biol 14: 1715-1721, 2017.

8. Salzman J, Gawad C, Wang PL, Lacayo N and Brown PO: Circular RNAs are the predominant transcript isoform from hundreds of human genes in diverse cell types. PLoS One 7: e30733, 2012

9. Liu J, Liu T, Wang $X$ and He A: Circles reshaping the RNA world: From waste to treasure. Mol Cancer 16: 58, 2017.

10. Hansen TB, Jensen TI, Clausen BH, Bramsen JB, Finsen B, Damgaard CK and Kjems J: Natural RNA circles function as efficient microRNA sponges. Nature 495: 384-388, 2013.

11. Han B, Chao J and Yao H: Circular RNA and its mechanisms in disease: From the bench to the clinic. Pharmacol Ther 187: 31-44. 2018.

12. Legnini I, Di Timoteo G, Rossi F, Morlando M, Briganti F, Sthandier O, Fatica A, Santini T, Andronache A, Wade M, et al: Circ-ZNF609 is a circular RNA that can be translated and functions in myogenesis. Mol Cell 66: 22-37, 2017.

13. Kristensen LS, Hansen TB, Veno MT and Kjems J: Circular RNAs in cancer: Opportunities and challenges in the field. Oncogene 37: 555-565, 2017.

14. Fan X, Weng X, Zhao Y, Chen W, Gan T and Xu D: Circular RNAs in cardiovascular disease: An overview. Biomed Res Int 2017: 5135781, 2017.

15. Floris G, Zhang L, Follesa P and Sun T: Regulatory role of circular RNAs and neurological disorders. Mol Neurobiol 54: 5156-5165, 2017.

16. Shan K, Liu C, Liu BH, Chen X, Dong R, Liu X, Zhang YY, Liu B, Zhang SJ, Wang JJ, et al: Circular noncoding RNA HIPK3 mediates retinal vascular dysfunction in diabetes mellitus. Circulation 136: 1629-1642, 2017.

17. Ciuclan L, Bonneau O, Hussey M, Duggan N, Holmes AM, Good R, Stringer R, Jones P, Morrell NW, Jarai G, et al: A novel murine model of severe pulmonary arterial hypertension. Am J Respir Crit Care Med 184: 1171-1182, 2011.

18. Livak KJ and Schmittgen TD: Analysis of relative gene expression data using real-time quantitative PCR and the $2^{-\Delta \Delta C T}$ method. Methods 25: 402-408, 2001.

19. Wang Y, Mo Y, Gong Z, Yang X, Yang M, Zhang S, Xiong F, Xiang B, Zhou M, Liao Q, et al: Circular RNAs in human cancer. Mol Cancer 16: 25, 2017. 
20. Enright AJ, John B, Gaul U, Tuschl T, Sander C and Marks DS: MicroRNA targets in Drosophila. Genome Biol 5: R1, 2003.

21. Pasquinelli AE: MicroRNAs and their targets: Recognition, regulation and an emerging reciprocal relationship. Nat Rev Genet 13: 271-282, 2012.

22. Dweep H and Gretz N: miRWalk2.0: A comprehensive atlas of microRNA-target interactions. Nat Methods 12: 697, 2015.

23. Vlachos IS, Zagganas K, Paraskevopoulou MD, Georgakilas G, Karagkouni D, Vergoulis T, Dalamagas T and Hatzigeorgiou AG: DIANA-miRPath v3.0: Deciphering microRNA function with experimental support. Nucleic Acids Res 43: W460-W466, 2015.

24. Wang W, Liu J, Ma A, Miao R, Jin Y, Zhang H, Xu K, Wang C and Wang J: mTORC1 is involved in hypoxia-induced pulmonary hypertension through the activation of Notch3. J Cell Physiol 229: 2117-2125, 2014.

25. Kan M, Shumyatcher M and Himes BE: Using omics approaches to understand pulmonary diseases. Respir Res 18: 149, 2017.

26. Bienertova-Vasku J, Novak J and Vasku A: MicroRNAs in pulmonary arterial hypertension: Pathogenesis, diagnosis and treatment. J Am Soc Hypertens 9: 221-234, 2015.

27. Deng L, Bradshaw AC and Baker AH: Role of noncoding RNA in vascular remodelling. Curr Opin Lipidol 27: 439-448, 2016.

28. Yao JT, Zhao SH, Liu QP, Lv MQ, Zhou DX, Liao ZJ and Nan KJ: Over-expression of CircRNA_100876 in non-small cell lung cancer and its prognostic value. Pathol Res Pract 213: 453-456, 2017.

29. Zhu X, Wang X, Wei S, Chen Y, Chen Y, Fan X, Han S and Wu G: hsa_circ_0013958: A circular RNA and potential novel biomarker for lung adenocarcinoma. FEBS J 284: 2170-2182, 2017.

30. Zhuang ZG, Zhang JA, Luo HL, Liu GB, Lu YB, Ge NH, Zheng BY, Li RX, Chen C, Wang X, et al: The circular RNA of peripheral blood mononuclear cells: Hsa_circ_0005836 as a new diagnostic biomarker and therapeutic target of active pulmonary tuberculosis. Mol Immunol 90: 264-272, 2017.

31. Wan QQ, Wu D and Ye QF: The expression profiles of circRNAs in lung tissues from rats with lipopolysaccharide-induced acute respiratory distress syndrome: A microarray study. Biochem Biophys Res Commun 493: 684-689, 2017.

32. Miao R, Wang Y, Wan J, Leng D, Gong J, Li J, Liang Y, Zhai Z and Yang Y: Microarray expression profile of circular RNAs in chronic thromboembolic pulmonary hypertension. Medicine 96 e7354, 2017.

33. Wu Y, Huang A, Li T, Su X, Ding H, Li H, Qin X, Hou L, Zhao Q, Ge X, et al: MiR-152 reduces human umbilical vein endothelial cell proliferation and migration by targeting ADAM17. Febs Lett 588: 2063-2069, 2014.

34. Liu X, Li J, Qin F and Dai S: miR-152 as a tumor suppressor microRNA: Target recognition and regulation in cancer. Oncol Lett 11: 3911-3916, 2016.

35. Nie L, Zhao YB, Pan JL, Lei, Y, Liu M, Long Y, Zhang JH, Hu Y, Xu MQ, Yuan DZ and Yue LM: Progesterone-induced miR-152 inhibits the proliferation of endometrial epithelial cells by downregulating WNT-1. Reprod Sci 24: 1444-1453, 2017.

36. Ge S, Wang D, Kong Q, Gao W and Sun J: Function of miR-152 as a tumor suppressor in human breast cancer by targeting PIK3CA. Oncol Res 25: 1363-1371, 2017.
37. Sun J, Tian X, Zhang J, Huang Y, Lin X, Chen L and Zhang S: Regulation of human glioma cell apoptosis and invasion by miR-152-3p through targeting DNMT1 and regulating NF2: MiR-152-3p regulate glioma cell apoptosis and invasion. J Exp Clin Cancer Res 36: 100, 2017.

38. Wang S, Wang L, Dou L, Guo J, Fang W, Li M, Meng X, Man Y, Shen T, Huang X and Li J: MicroRNA 152 regulates hepatic glycogenesis by targeting PTEN. FEBS J 283: 1935-1946, 2016.

39. Gu S, Ran S, Liu B and Liang J: miR-152 induces human dental pulp stem cell senescence by inhibiting SIRT7 expression. FEBS Lett 590: 1123-1131, 2016.

40. Tao J, Liu W, Shang G, Zheng Y, Huang J, Lin R and Chen L: MiR-207/352 regulate lysosomal-associated membrane proteins and enzymes following ischemic stroke. Neuroscience 305: 1-14, 2015.

41. Lu X, Lv S, Mi Y, Wang L and Wang G: Neuroprotective effect of miR-665 against sevoflurane anesthesia-induced cognitive dysfunction in rats through PI3K/Akt signaling pathway by targeting insulin-like growth factor 2 . Am J Transl Res 9: 1344-1356, 2017

42. Sehgal PB and Mukhopadhyay S: Pulmonary arterial hypertension: A disease of tethers, SNAREs and SNAPs? Am J Physio Heart Circ Physiol 293: H77-H85, 2007.

43. Fulton D, Li X, Bordan Z, Haigh S, Bentley A, Chen F and Barman SA: Reactive oxygen and nitrogen species in the development of pulmonary hypertension. Antioxidants 6: E54, 2017.

44. Thenappan $\mathrm{T}$ and Weir EK: The nitric oxide pathway-A potential target for precision medicine in pulmonary arterial hypertension. Am J Cardiol 120: S69-S70, 2017.

45. Zhang C, Ma C, Yao H, Zhang L, Yu X, Liu Y, Shen T, Zhang L, Zhang F, Chen $X$ and Zhu D: 12-Lipoxygenase and 12-hydroxyeicosatetraenoic acid regulate hypoxic angiogenesis and survival of pulmonary artery endothelial cells via PI3K/Akt pathway. Am J Physiol Lung Cell Mol Physiol 314: L606-L616, 2018.

46. Xia XD, Lee J, Khan S, Ye L, Li Y and Dong L: Suppression of phosphatidylinositol 3-kinase/Akt signaling attenuates hypoxia-induced pulmonary hypertension through the downregulation of lysyl oxidase. DNA Cell Biol 35: 599-606, 2016.

47. Lim CS, Kiriakidis S, Sandison A, Paleolog EM and Davies AH: Hypoxia-inducible factor pathway and diseases of the vascular wall. J Vasc Surg 58: 219-230, 2013.

48. Awad KS, West JD, de Jesus PV and MacLean M: Novel signaling pathways in pulmonary arterial hypertension (2015 Grover conference series). Pulm Circ 6: 285-294, 2016.

49. Wilusz JE: A $360^{\circ}$ view of circular RNAs: From biogenesis to functions. WIREs RNA e1478, 2018.

This work is licensed under a Creative Commons Attribution-NonCommercial-NoDerivatives 4.0 International (CC BY-NC-ND 4.0) License. 